\title{
ABDOMEN AGUDO EN PACIENTES CON VIH/SIDA ATENDIDOS EN UN HOSPITAL NACIONAL DE LIMA, PERÚ
}

\author{
Leonor Montoya ${ }^{1, a}$, Ericka Rodríguez ${ }^{1, a}$, Grace Zúñiga1,a, Gaby Yamamoto²,b Elsa González ${ }^{3, a}$
}

\begin{abstract}
RESUMEN
El objetivo del estudio fue describir las características en la presentación y el manejo del abdomen agudo (AA) en pacientes con el virus de la inmunodeficiencia humana $(\mathrm{VIH})$. Se revisaron las historias clínicas de 97 pacientes portadores del VIH que cursaron con un cuadro de AA y que fueron atendidos en el período 2006-2011 en el Hospital Nacional Cayetano Heredia en Lima, Perú. Se encontró que el 1,6\% de los pacientes inmunosuprimidos ingresó a sala de operaciones. La apendicectomía fue el procedimiento quirúrgico más frecuentemente (33,3\%). La morbilidad fue de $28,1 \%$, y la mortalidad posoperatoria de 9,4\%. La infección por Mycobacterium tuberculosis fue la causa más común de dolor abdominal agudo con el $26,8 \%$. Los datos sugieren que una pronta decisión quirúrgica frente a un cuadro compatible con AA en pacientes con VIH podría evitar una importante morbimortalidad.
\end{abstract}

Palabras clave: Abdomen agudo; VIH; Síndrome de inmunodeficiencia adquirida (fuente: DeCS BIREME).

\section{ACUTE ABDOMEN IN PATIENTS WITH HIV/AIDS SEEN IN A NATIONAL HOSPITAL OF LIMA, PERU}

\begin{abstract}
The aim of this study was to describe the features in the presentation and management of acute abdomen (AA) in patients with human immunodeficiency virus (HIV). We reviewed the medical records of $97 \mathrm{HIV}$ patients who presented with AA and were seen in 2006-2011 at Cayetano Heredia National Hospital in Lima, Peru. 1.6\% of immunosuppressed patients underwent surgery. Appendectomy was the most common surgical procedure (33.3\%). Morbidity was $28.1 \%$ and postoperative mortality was $9.4 \%$. Infection by mycobacterium tuberculosis was the most common cause of acute abdominal pain, at $26.8 \%$. Data suggest that an early surgical decision for cases of AA in HIV patients may prevent significant morbidity and mortality.
\end{abstract}

Key words: Abdomen, acute; HIV; Acquired immunodeficiency syndrome (source: MeSH NLM).

\section{INTRODUCCIÓN}

El VIH es un problema sanitario mundial, más de 35 millones de personas están infectadas a pesar de las campañas informativas y políticas de prevención existentes ${ }^{(1)}$. En Perú, se notificaron 52053 casos de VIH y 31018 del síndrome de inmunodeficiencia adquirida (SIDA) (2). Por tanto, cada vez más profesionales de salud tratarán pacientes seropositivos, entonces resulta necesario conocer el manejo de los problemas que surgen en ellos, como el AA, que implica la aparición repentina de síntomas abdominales graves que ponen en riesgo la vida. El dolor abdominal es la manifestación más común y la de mayor reto diagnóstico y terapéutico (3).

Establecer un diagnóstico certero, identificando patologías abdominales que requieren manejo quirúrgico, diferenciándolas de aquellas que requieren tratamiento médico, es fundamental para orientar la conducta terapéutica. En pacientes con $\mathrm{VIH}$ es necesario considerar entre los diagnósticos diferenciales, enfermedades poco comunes en la población general como infecciones oportunistas (IO) y neoplasias malignas $(\mathrm{NM}){ }^{(1)}$. Entre las enfermedades

\footnotetext{
Facultad de Medicina. Universidad Peruana Cayetano Heredia. Lima, Perú.

Hospital Nacional Cayetano Heredia. Lima, Perú.

Instituto de Medicina Tropical "Alexander von Humboldt". Lima, Perú.

Médico cirujano; ${ }^{\text {b cirujano general }}$

Recibido: : 07-03-14 Aprobado: 23-07-14
}

Citar como: Montoya L, Rodríguez E, Zúñiga G, Yamamoto G, González E. Abdomen agudo en pacientes con VIH/Sida atendidos en un hospital nacional de Lima, Perú. Rev Peru Med Exp Salud Publica. 2014;31(3):515-19. 
más frecuentes asociadas al VIH en el Perú tenemos a la infección por citomegalovirus (CMV), sarcoma de Kaposi, linfoma no Hodgkin e infecciones por micobacterias como la tuberculosis ${ }^{(4,5)}$.

Los pacientes seropositivos pueden cursar con un AA no relacionado al $\mathrm{VIH}$, como apendicitis y colecistitis, con incidencia similar a la población general. La apendicectomía ha sido reportada como el procedimiento más común en diversos artículos ${ }^{(3,6)}$, pero en el más grande estudio del impacto del VIH/SIDA en la cirugía general, el $25,3 \%$ fue la cirugía anorrectal (7). La única investigación realizada en Perú del manejo quirúrgico del $\mathrm{AA}$ en pacientes seropositivos, fue previa a la implementación del TARGA ${ }^{(4)}$.

En este contexto, el objetivo es describir la presentación y el manejo del AA en pacientes con VIH/SIDA en el período 2006-2011 en el Hospital Nacional Cayetano Heredia $(\mathrm{HNCH})$, luego del inicio del tratamiento antirretroviral de gran actividad (TARGA).

\section{EL ESTUDIO}

Estudio descriptivo retrospectivo en pacientes mayores de 18 años, atendidos desde enero de 2006 a diciembre de 2011 en el HNCH en Lima, Perú. Se seleccionó pacientes con $\mathrm{VIH}$ según diagnósticos de egreso y códigos CIE-10 (B20.0-B24.X) que presentaron AA. Se descartaron 117 registros por criterios de exclusión.

Se revisaron 75 historias clínicas de pacientes vivos y 22 certificados de defunción. De las historias clínicas se extrajeron los siguientes datos: anamnesis, examen físico, reporte operatorio, exámenes de laboratorio e imagenológicos, evoluciones y terapéutica. De los certificados de defunción: fecha de nacimiento y fallecimiento, causa de muerte y los informes adjuntos en caso de indicación quirúrgica. Luego fueron transcritos a fichas de datos elaboradas con ítems específicos para interés del estudio.

Se tuvo acceso a la cohorte de VIH (COVIHS), programa al que ingresan pacientes seropositivos previo consentimiento informado, para obtener valores de CD4, carga viral (CV) y adherencia al TARGA. Los datos fueron ingresados a una hoja de cálculo $y$, posteriormente, analizados con el paquete estadístico Stata 12.

El protocolo del estudio fue aprobado por el Comité de Ética Institucional de la Universidad Peruana Cayetano Heredia y del $\mathrm{HNCH}$.

\section{HALLAZGOS}

Durante el período de estudio se atendieron 1895 pacientes con $\mathrm{VIH} / \mathrm{SIDA}, 97(5,1 \%)$ presentaron un cuadro de AA. El 1,6\% requirió intervención quirúrgica. La mediana de edad fue de 37 años (rango intercuartil: $18-64$ años) y 61 pacientes (62,9\%) pertenecían al sexo masculino. Sobre las características basales de pacientes vivos $(n=75)$ el $40,0 \%$ tuvo antecedente de medicación previa, entre sintomáticos y antibióticos, administrados por vía oral y sin prescripción médica. Aunque hubo otras comorbilidades, la tuberculosis fue el antecedente patológico más frecuente (32,0\%). El dolor abdominal estuvo presente en todos los pacientes representando el parámetro más importante de inclusión al estudio, seguido por náuseas y/o vómitos $(56,0 \%)$ y deposiciones líquidas (45,3\%) Tabla 1.

Las ecografías $(42,7 \%)$ y tomografías $(18,7 \%)$ fueron sugerentes de afección abdominal (apendicitis, colelitiasis, abscesos, etc.) en $61,3 \%$, y se encontró leucocitosis en $29,3 \%$. El $49,5 \%$ de vivos y fallecidos $(n=97)$ presentó manifestaciones clínicas e indicación quirúrgica según anamnesis; el 32,9\% necesitó cirugía de emergencia, mientras que el $16,5 \%$ requería cirugía electiva, sin embargo, solo el $30,9 \%$ de los pacientes se operó. Sobre el abordaje, se emplearon la laparoscopía y la laparotomía convencional, ambas, en $15,5 \%$ de los casos.

Respecto a las 30 intervenciones quirúrgicas realizadas, el 93,3\% fueron cirugías terapéuticas y 2 diagnósticas: una laparotomía exploratoria más biopsia de hígado, que resultó ser un adenocarcinoma hepático, y una laparoscopía diagnóstica más biopsia de peritoneo, compatible con tuberculosis. Ninguna cirugía fue reportada como innecesaria o sin alteraciones.

Tabla 1. Manifestaciones clínicas en pacientes con $\mathrm{VIH}$ que cursaron con patología compatible con síndrome abdominal agudo

\begin{tabular}{lcc}
\hline Manifestaciones & N & $(\%)$ \\
\hline Dolor abdominal & 75 & $(100)$ \\
Náuseas y vómitos & 42 & $(56,0)$ \\
\hline Deposiciones líquidas & 34 & $(45,3)$ \\
\hline Fiebre & 30 & $(40,0)$ \\
\hline Distensión abdominal & 24 & $(32,0)$ \\
\hline Signos peritoneales & 13 & $(17,3)$ \\
\multicolumn{1}{r}{ Mc Burney } & 3 & $(4,0)$ \\
\multicolumn{1}{r}{ Murphy } & 5 & $(6,6)$ \\
Rebote & 5 & $(6,6)$ \\
\hline Masa abdominal & 6 & $(8,0)$ \\
\hline \multicolumn{1}{r}{} & 4 & $(5,3)$ \\
\hline
\end{tabular}


Tabla 2. Procedimientos quirúrgicos realizados en pacientes con $\mathrm{VIH}$ y patología compatible con síndrome abdominal agudo

\begin{tabular}{|c|c|c|}
\hline Procedimiento & $\mathrm{N}=30$ & (\%) \\
\hline \multicolumn{3}{|l|}{ Apendicectomía } \\
\hline Laparoscópica & 4 & $(13,3)$ \\
\hline Abierta & 6 & $(20,0)$ \\
\hline Laparotomía exploratoria & 7 & $(23,3)$ \\
\hline Colecistectomía laparoscópica & 5 & $(16,7)$ \\
\hline Laparoscopía diagnóstica & 4 & $(13,3)$ \\
\hline Hernioplastía & 3 & $(9,9)$ \\
\hline $\begin{array}{l}\text { Histerosalpingooferectomía } \\
\text { bilateral }\end{array}$ & 1 & $(3,3)$ \\
\hline
\end{tabular}

Las enfermedades asociadas a inmunosupresión fueron la causa más común de dolor abdominal, liderando la tuberculosis con $26,8 \%$, en sus variantes gastrointestinal $(14,4 \%)$ y multisistémica (12,4\%). Las patologías quirúrgicas más frecuentes fueron las colecistopatías $(20,6 \%)$ y las apendicopatías $(13,4 \%)$, hubo una apendicitis por CMV (Tabla 2).

Tabla 2. Etiología del abdomen agudo en pacientes con VIH

\begin{tabular}{|c|c|c|}
\hline Etiología & $\mathrm{N}=97$ & $(\%)$ \\
\hline \multicolumn{3}{|l|}{ Tuberculosis } \\
\hline Gastrointestinal & 14 & $(14,4)$ \\
\hline Multisistémica & 12 & $(12,4)$ \\
\hline \multicolumn{3}{|l|}{ Colecistopatías } \\
\hline Colecistitis aguda litiásica & 16 & $(16,5)$ \\
\hline c/ Coledocolitiasis & 2 & $(12,5)$ \\
\hline c/ Piocolecisto & 2 & $(12,5)$ \\
\hline Pancreatitis aguda biliar & 3 & $(3,1)$ \\
\hline Neoplasia maligna: insulinoma & 1 & $(1,0)$ \\
\hline \multicolumn{3}{|l|}{ Gastroenterocolitis agudas } \\
\hline $\mathrm{p} /$ Salmonella $s p$ & 1 & $(1,0)$ \\
\hline p/ Coccídeas & 1 & $(1,0)$ \\
\hline $\mathrm{p} /$ Isospora belli & 1 & $(1,0)$ \\
\hline $\mathrm{p} /$ Cryptosporidium $s p$ & 1 & $(1,0)$ \\
\hline EAD & 10 & $(10,3)$ \\
\hline \multicolumn{3}{|l|}{ Apendicopatías } \\
\hline Apendicitis aguda & 11 & $(11,3)$ \\
\hline $\mathrm{p} / \mathrm{CMV}$ & 1 & $(1,0)$ \\
\hline c/ Adenitis mesentérica & 1 & $(1,0)$ \\
\hline c/ Peritonitis localizada & 3 & $(3,1)$ \\
\hline Plastrón apendicular & 2 & $(2,1)$ \\
\hline Hepatopatías & 4 & $(4,1)$ \\
\hline Patologías anorrectales & 4 & $(4,1)$ \\
\hline Patologías ginecológicas & 3 & $(3,1)$ \\
\hline Suboclusión Intestinal & 2 & $(2,1)$ \\
\hline Hernias & 3 & $(3,1)$ \\
\hline Otros* & 8 & $(8,3)$ \\
\hline
\end{tabular}

*Incluye ileítis (1), pancreatitis aguda no biliar (3), hemoperitoneo postrauma (1), diverticulitis (1), sarcoma de Kaposi (1), linfoma de células grandes (1)
Hubo complicaciones en $28,1 \%$ de los sometidos a cirugía: síndrome febril $(13,3 \%)$, íleo adinámico (3,3\%), sangrado de herida operatoria $(3,3 \%)$, neumonía intrahospitalaria $(6,7 \%)$ e insuficiencia renal aguda de tipo prerrenal (3,3\%). Tres pacientes $(10,0 \%)$ fallecieron, el primero tras una apendicectomía convencional, desarrolló una fístula enterocutánea que prolongó su internamiento, murió por neumonía intrahospitalaria; el segundo fue sometido a resección y desbridamiento del páncreas, luego reintervenido para anastomosis pancreatoduodenal y pancreatectomía subtotal distal, pero no sobrevivió (no hay detalles sobre deceso). El último paciente presentó una hemorragia digestiva alta por lesión vascular, tras colecistectomía laparoscópica, que devino en shock hipovolémico y muerte.

Se extrajeron cálculos por colangiopancreatografía retrógrada endoscópica (CPRE) en 4,1\% de los casos, se drenaron abscesos hepáticos por radiología intervencionista en $3,1 \%$, y en $9,3 \%$ de los casos se realizaron biopsias. El diagnóstico reciente de $\mathrm{VIH}$, durante la hospitalización, correspondió a 20 de 77 pacientes con información disponible (25,9\%). El 54,5\% recibían tratamiento antirretroviral y $85,7 \%$ de estos, tenían adherencia mayor a 95\%. Sobre el recuento de CD4 y CV se obtuvo datos correspondientes a la fecha más próxima del cuadro abdominal importante para la investigación, solo en 69 pacientes. Hubo diferencias entre los grupos de pacientes vivos y fallecidos: En el primero (56 casos), la mediana de CD4=187,5 cél/mL (rango intercuartil 5-788 cél $/ \mathrm{mL}$ ) y el $51,8 \%$ tuvo un recuento de CD4<200 cél/mL, siendo la media $254,5 \mathrm{cél} / \mathrm{mL}$ y, la mediana de $C V=400$ copias $/ \mathrm{mm}^{3}$. En el segundo grupo (13 casos), la mediana del recuento de CD4=43 cél/mL (rango intercuartil 4-434 cél $/ \mathrm{mL}$ ), el $76,9 \%$ de los pacientes presentó valores $<200$ cél $/ \mathrm{mL}$, siendo la media $106,5 \mathrm{cél} / \mathrm{mL}$ y la mediana de $\mathrm{CV}=4583$ copias $/ \mathrm{mm}^{3}$.

\section{DISCUSIÓN}

El abordaje del AA en pacientes seropositivos resulta complejo por la etiología diversa y las manifestaciones clínicas poco específicas. Si bien existen causas asociadas a la inmunosupresión, las patologías de causa quirúrgica tienen similar incidencia a las reportadas en la población general ${ }^{(8)}$. En el presente estudio el 1,6\% de los pacientes con $\mathrm{VIH}$ requirió intervención quirúrgica por $A A$, resultado similar a un estudio previo donde el requerimiento de cirugía fue $1,8 \%$.

Se evidenció una alta frecuencia de infecciones relacionada a la inmunosupresión, siendo la más frecuente la tuberculosis, resultado esperado debido a la alta incidencia de esta infección en nuestro medio. 
Además, se encontró un caso de sarcoma de Kaposi, que según la literatura, es el cáncer más relacionado con VIH/SIDA, aunque con la aparición del TARGA su incidencia ha disminuido, y la supervivencia a 5 años ha alcanzado $90 \%{ }^{(12)}$; asimismo, se halló un caso de linfoma no Hodking, que está presente 20-50 veces más en inmunosuprimidos por lo que es la segunda NM más frecuentemente asociada con el VIH ${ }^{(12)}$. El estudio imagenológico fue, probablemente, el más útil como ayuda diagnóstica, ya que fue determinante en la decisión de ingreso a SOP. Menos de 1/3 de los pacientes presentaron leucocitosis, quizás por su inmunosupresión basal. Aunque la literatura describe la realización de cirugías innecesarias (sin patología específica) hasta en $26,1 \%{ }^{(4)}$, en este estudio no se reporta ninguna, lo que representaría un avance en la experticia diagnóstica.

Siempre que sea posible debe procurarse un tratamiento mínimamente invasivo, tal como sugiere la literatura ${ }^{(9)}$. En el presente estudio se reporta un mayor número de laparoscopías realizadas, diferente a intervenciones previas a la era TARGA (4), donde solo dos casos fueron manejados con dicha técnica. Se puede evidenciar, entonces, una notable mejoría respecto al uso del laparoscopio en el $\mathrm{HNCH}$. Además, se realizaron CPRE, toma de biopsias y drenaje de abscesos con guía radiológica. Todo, con la finalidad de evitar cirugías innecesarias y disminuir el riesgo de transmisión de VIH ${ }^{(10)}$. La apendicectomía fue la cirugía más realizada, similar a lo reportado en artículos extranjeros, como el estudio prospectivo de Smith en Sudáfrica ${ }^{(17)}$. Sin embargo, de haberse llevado a cabo todas las colecistectomías programadas, hubieran ocupado el primer lugar. Probablemente, la falta de control ambulatorio para programación de cirugía electiva y la discontinuidad de los pacientes al servicio de cirugía general, fueron causa de la no realización de las colecistectomías.

Debería solicitarse análisis anatomopatológicos en todos los casos, pues los cuadros apendiculares y vesiculares podrían tener patrones etiológicos característicos del deficiente estado inmunológico de los pacientes ${ }^{(11)}$. En el presente trabajo solo se reporta cinco histopatologías, lo que refleja una capacidad diagnóstica restringida. Se notifican tres casos de pancreatitis aguda biliar, contrario a lo encontrado en la literatura, que señala su alta incidencia en pacientes seropositivos ${ }^{(9)}$ pero solo uno recibía TARGA al momento del diagnóstico. Se conocen casos de pancreatitis medicamentosa asociada con dichos antivirales ${ }^{(8)}$. Hubo tres pacientes fallecidos por complicaciones post quirúrgicas. Una causa básica de muerte importante, hasta en $50 \%$, fue la infección por micobacterias, un paciente cursó con colitis infecciosa por Mycobacterium avium, infección que puede diseminarse si no se recibe quimioprofilaxis dentro de los dos primeros años posteriores al diagnóstico de SIDA (13); otro presentó un absceso del psoas, complicación por continuidad de la infección por Mycobacterium tuberculosis a la columna vertebral, frecuente en nuestro medio hasta en un 50 a $60 \%$ de las formas osteoarticulares ${ }^{(14)}$.

Aunque actualmente más pacientes son conscientes de su enfermedad y de la importancia de la prevención de complicaciones, casi $1 / 3$ de los pacientes conocieron su seroconversión recién durante la hospitalización de interés y solo un poco más de la mitad de ellos recibían TARGA. Además, la mitad de los pacientes, con o sin tratamiento antirretroviral, tuvieron un conteo de CD4<200 cél/mL y todos presentaron CV detectable, lo que refleja un pobre estado inmune, predisponente a IO y NM.

La principal limitación del estudio, fue el inadecuado almacenaje de documentos en el $\mathrm{HNCH}$. Un importante número de registros $(6,2 \%$ del total de pacientes seropositivos) fue separado del estudio por pérdida, parcial o completa de las historias clínicas, errores de digitación de la base de datos y el precario almacenaje de certificados de defunción.

Concluimos que debe prestarse especial atención al paciente con VIH que presente patología compatible con AA, debido al grado de dificultad diagnóstica por sus múltiples posibilidades etiológicas, para discernir oportunamente si se requiere manejo quirúrgico. Sin embargo, a pesar que el ingreso a SOP tiene un papel importante en el tratamiento integral del $A A$, el diagnóstico diferencial más importante es la tuberculosis y que puede ser manejada médicamente con óptimos resultados, disminuyendo los riesgos que conlleva una cirugía innecesaria.

Contribuciones de autoría: LM, ER y GZ han participado en la concepción y el diseño del artículo, recolección y obtención de datos, GY y EV trabajaron en el análisis e interpretación de los resultados, así como en la redacción del artículo. GY y EG revisaron de manera crítica el artículo y aprobaron la versión final. EG fue la principal asesora estadística, y GY realizó la asesoría técnica en presente trabajo de investigación.

Fuentes de financiamiento: el presente estudio ha sido autofinanciado. No se han recibido donaciones ni apoyo económico de ningún tipo.

Conflicto de interés: no existe conflicto de intereses, ni económico, ni institucional, laboral o personal por parte de ninguno de los autores. 


\section{REFERENCIAS BIBLIOGRÁFICAS}

1. Centers for Disease Control and Prevention. National Center for HIV/AIDS, Viral Hepatitis, Sexual Transmitted Diseases and Tuberculosis Prevention [Internet]. Atlanta: CDC; 2012 [citado el 5 de enero del 2014]. Disponible en: http://www.cdc.gov/ nchhstp/

2. Perú, Ministerio de Salud. Situación del VIH/SIDA en el Perú. Boletín Epidemiológico Mensual [Internet]. Lima: MINSA; 2013 [citado el 5 de enero del 2014]. Disponible en: http:// www.app.minsa.gob.pe/bsc/detalle indbsc. asp ? lcind $=18 \&$ lcobj $=4 \&$ lcper $=1 \& \mathrm{lcfreg}=10 / 2 / 2014$

3. Kosmidis Ch, Anthimidis G, Vasiliadou K, Interbalkan European Medical Center, Department of Surgery. Acute Abdomen and HIV Infection. Athens: INTECH; 2011.

4. Martínez F, Rodríguez M, Samalvides F. Abdomen agudo quirúrgico en pacientes infectados con el virus de inmunodeficiencia humana en el Hospital Nacional Cayetano Heredia. Rev Med. Hered. 2004 OctDic;15(4):188-96.

5. Yriberri $S$, Cervera Z, Soriano C, Frisancho O, Machado A. Tuberculosis digestiva en el Hospital Nacional Edgardo Rebagliati Martins (HNERM): Un estudio retrospectivo de 5 años (1993-1998). Rev Gastroenterol Peru. 1998 SetDic;18(3):238-49.

6. Foo E1, Sim R, Lim HY, Chan ST, Leo YS, Wong SY. Abdominal surgery in human immunodeficiency virus (HIV) infected patients--early local experience. Ann Acad Med Singapore. 1998 Nov;27(6):759-62.

7. RS Dua, SA Wajed, MC Winslet. Impact of HIV and AIDS on surgical practice. Ann R Coll Surg Engl. 2007 May;89(4):354-8.

8. Ferat-Osorio E, Guzmán-Valdivia G, Rosales L, Treviño-Pérez S, Nieto-Cisneros L, Majluf-Cruz A. Características clínicas y evolución de pacientes con SIDA y dolor abdominal agudo. Gac Med Mex. 2005 SetOct;141(5):357-62.

9. Ramírez M, Braghetto I. VIH y cirugía. Boletines FELAC. 2009;14(2).

10. Tanner AG, Hartley JE, Darzi A, Rosin RD, Monson JR. Laparoscopic surgery in patients with human immunodeficiency virus. Br J Surg. 1994 Nov;81(11):1647-8.

11. Whitney TM, Brunel W, Russell TR, Bossart KJ, Schecter WP. Emergent abdominal surgery in AIDS: experience in San Francisco. Am J Surg. 1994 Sep;168(3):239-43.

12. American Society of Clinical Oncology. HIV and AIDS Related to Cancer [Internet]. Alexandria: ASCO; 2013 [citado el 5 de enero del 2014]. Disponible en: http://www.cancer. net/about-us

13. American Society of Clinical Oncology. HIV and AIDS Related to Cancer: Statistics [Internet]. Alexandria: ASCO; 2013 [citado el 5 de enero del
2014]. Disponible en: http://www. cancer.net/cancer-types/hiv-and-aidsrelated-cancer/statistics

14. Nightingale SD, Byrd LT, Southern PM, Jockusch JD, Cal SX, Wynne BA. Incidence of Mycobacterium aviumintracellulare complex bacteremia in human immunodeficiency viruspositive patients. J Infect Dis. 1992 Jun;165(6):1082-5.

15. Laube I, Pfyffer GE, Kronauer CM. [Extrapulmonary infections with Mycobacterium tuberculosis complex]. Schweiz Med Wochenschr. 1996 Nov 30;126(48):2068-71. [Article in German]

16. Saltzman DJ, Williams RA, Gelfand DV, Wilson SE. The surgeon and AIDS: twenty years later. Arch Surg. 2005 Oct;140(10):961-7.

17. Lowy AM, Barie PS. Laparotomy in patients infected with human immunodeficiency virus: indications and outcome. Br J Surg. 1994 Jul;81(7):942-5.

18. Smith SJ, Du Toit RS. The acute AIDS abdomen - a prospective clinical and pathological study. S Afr J Surg. 2005 Aug;43(3):88.

Correspondencia: Leonor Montoya Montenegro

Dirección: Av. Abraham Lincoln 461, Lima 22, Lima, Perú.

Teléfono: +51964936980

Correo electrónico:leonor.montoya@upch.pe

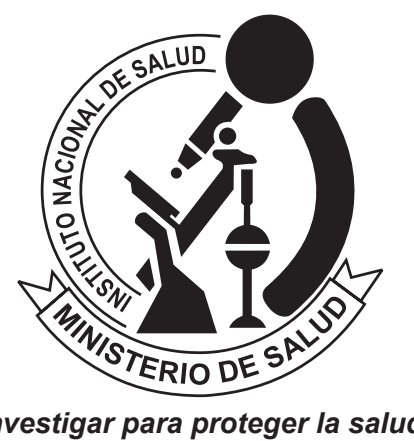

Investigar para proteger la salud

\section{REVISTA PERUANA DE MEDICINA EXPERIMENTAL Y SALUD PÚBLICA CUMPLIENDO SUS METAS Y PROYECTÁNDOSE AL FUTURO}

\section{Visite los contenidos de la revista en: www.ins.gob.pe/rpmesp}

